\title{
A Modified Under-Vastus Approach for Knee Arthroplasty with Anatomical Repair of Soft Tissue
}

\author{
Takafumi Hiranaka, MD, Toshikazu Tanaka, MD, Takaaki Fujishiro, MD, \\ Kensuke Anjiki, MD, Naosuke Nagata, MD, Daiya Kitazawa, MD, Ken Kotoura, MD, Koji Okamoto, MD
}

Department of Orthopaedic Surgery and Joint Surgery Centre, Takatsuki General Hospital, Osaka, Japan

\begin{abstract}
The under-vastus approach (UVA) is a modification of the conventional subvastus approach for knee arthroplasty. Our modified UVA allows the muscles to be spared while offering good exposure of the operative field and facilitating anatomical repair of the capsule and fascia. A medial parapatellar incision is made and the vastus medialis fascia is incised along the same line. The muscle belly is not incised but detached from the surrounding fascia and retracted laterally. The suprapatellar pouch capsule is then laterally incised under the vastus muscles. The patella can then be easily retracted laterally and adequate exposure is possible. After the procedure, both capsule and fascia can be anatomically repaired. We use this approach for all primary arthroplasties in our practice. There has been no case in which an additional muscle incision was required, and there were no intraoperative complications. The modified UVA allows for excellent exposure of the operative field as well as muscle retention in both primary total and unicompartmental knee arthroplasties.
\end{abstract}

Keywords: Knee, Osteoarthritis, Arthroplasty

The subvastus approach (SVA) for total knee arthroplasty was first reported by Hofmann et al. ${ }^{1)}$ in 1991. This approach does not require an incision of the vastus medialis (VM), and its reported advantages include reduced pain, early functional recovery, and less frequent lateral retinacular release, as well as preservation of the patellar blood supply. ${ }^{2)}$

In the SVA, including the minimally invasive SVA, ${ }^{3)}$ and the so-called quadriceps-sparing approach with a commercially available instrument set, ${ }^{4)}$ the capsulotomy is performed in a reversed L shape where the transverse cut is made medially along the lower border of the VM (Fig. 1A). ${ }^{5}$ With these approaches, the capsule of the suprapatellar pouch remains intact and tensile, eventually prohibiting the lateral movement of the patella.

Received July 22, 2019; Accepted September 16, 2019

Correspondence to: Takafumi Hiranaka, MD

Department of Orthopaedic Surgery and Joint Surgery Centre, Takatsuki

General Hospital, 1-3-13, Kosobe, Takatsuki, Osaka 5691192, Japan

Tel: +81-72-6813801, Fax: +81-72-6823834

E-mail: takafumi.hiranaka@gmail.com
Tatsumi $^{6)}$ modified the SVA for better exposure of the operative field. In his approach, the suprapatellar pouch is incised at the midline under the distal end of the VM. He named this approach the under-vastus approach (UVA) and reported that it could be performed easily and provide good exposure. Through this approach, the fascia of the VM is detached from the surrounding capsule and periosteum and retracted laterally along with the muscle belly. Although the lateralized incision of the suprapatellar pouch capsule makes lateralization of the patella easier, the detachment of the fascia is not always easy (Fig. 1B). Fascia is sometimes torn and a thickened fascia can be an obstacle to patellar lateralization. Moreover, no report has mentioned on capsular repair. If the capsule is repaired completely, intra-articular haemorrhage from the joint cavity will not occur (hemorrhage can cause swelling and inflammation of the thigh).

To make it more universal, easy to perform, and less invasive, we modified the UVA as follows: (1) Incise the fascia and laterally retract only the muscle belly of the VM. (2) Incise the capsule more laterally under the VM. (3) Repair the capsule in a complete and watertight manner (Fig. 
Hiranaka et al. Modified Under-Vastus Approach for Knee Arthroplasty

Clinics in Orthopedic Surgery • Vol. 11, No. 4, 2019•www.ecios.org

(A) Subvastus

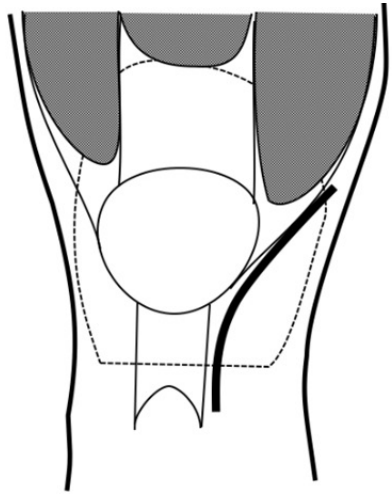

(B) Original under-vastus

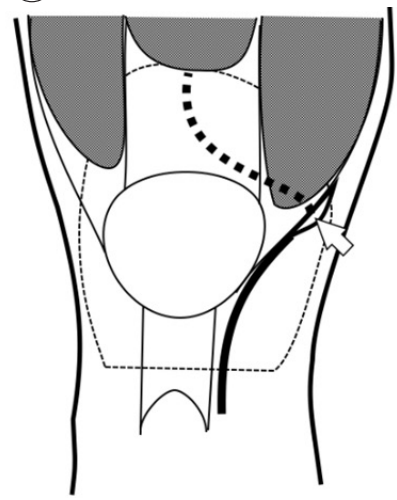

(C) Modified under-vastus

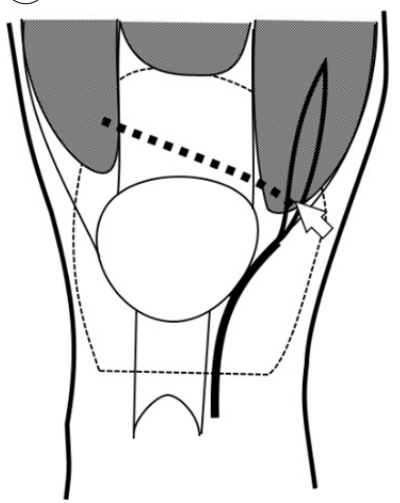

Fig. 1. Line drawing showing the capsule and fascia incisions in three different approaches. Solid lines indicate capsular incision medial to the patella and patellar tendon. The dotted lines indicate capsular incision of suprapatellar pouch under the quadriceps muscle. The arrows indicate where the detachment starts. (A) The conventional subvastus approach. The capsule is cut along the medial border of the patellar tendon and the lower border of the vastus medialis, forming a reversed $L$ shape. (B) The under-vastus approach devised by Tatsumil). The fascia at the distal end of the vastus medialis is detached and retracted laterally. The capsule of the suprapatellar pouch is incised at the midline. (C) Our modified under-vastus approach. The fascia is incised longitudinally and the muscle belly is retracted laterally. Then, the capsule of the suprapatellar pouch is incised far laterally.
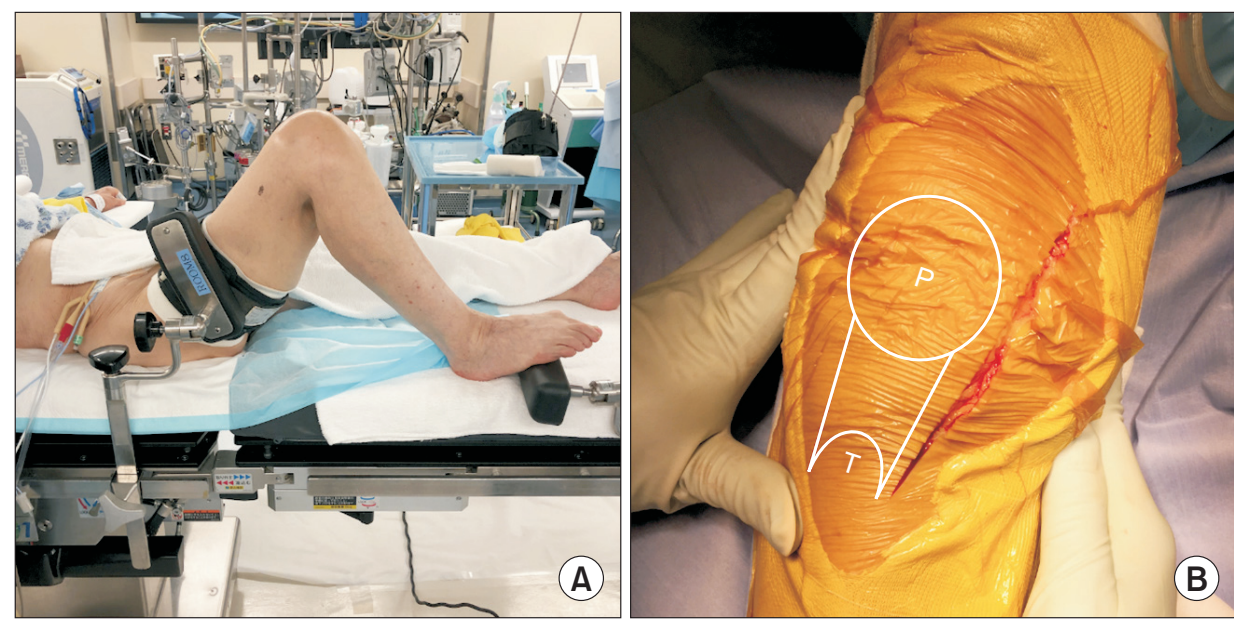

Fig. 2. Leg positioning and skin incision. (A) The operated leg is placed on the table with two-leg supports to maintain the knee flexed to $100^{\circ}-110^{\circ}$. (B) In the anteromedial aspect of the operated knee, the skin incision is made, starting from 2-3 $\mathrm{cm}$ proximal to the superior pole of the patella (P), passing 1-2 $\mathrm{cm}$ medial to the patella and the patellar tendon, and ending just medial to the tibial tuberosity (T).
1C). We have used this approach for all primary knee arthroplasties (both unicompartmental and total) uneventfully without difficulty. This technical note describes the details of our modified UVA.

\section{TECHNIQUE}

\section{Setting}

The procedure is performed under general or spinal anaesthesia. Two-leg supports are used to stabilize the knee flexed to $100^{\circ}-110^{\circ}$ on the operative table (Fig. $2 \mathrm{~A}$ ). The procedure is carried out with the knee in extension, flexion, and maximum flexion depending on the stage of surgery. A written informed consent was obtainded from the patient.

\section{Incision}

An anteromedial skin incision is made from $2-3 \mathrm{~cm}$ proximal and 1-2 cm medial to the patella and descends parallel to the medial border of the patella and the patellar tendon, ending just medial to the tibial tuberosity (Fig. 2B) with the knee in the flexion position. The patellar tendon is then identified and a short capsular cut is made just medial to it. The knee is then extended and the subcutaneous tissues are detached from the underlying quadriceps fascia. In elderly female patients, the fascia can be extremely thin and may adhere to the subcutaneous tissue. Care must be taken not to damage the fascia.

\section{Fasciotomy and Capsulotomy}

In the SVA, the capsule is cut just below the VM muscle. 
Hiranaka et al. Modified Under-Vastus Approach for Knee Arthroplasty

Clinics in Orthopedic Surgery • Vol. 11, No. 4, 2019•www.ecios.org

In the Tatsumi's UVA, the fascia is detached from surrounding tissues. By contrast, in the modified UVA, the fascia is cut along the same line as the skin incision. The muscle belly is not incised but gently detached from the fascia. The detachment starts from the medial position and continues laterally beneath the muscle as far as to the lateral side of the femur (Fig. 3A). A periosteum elevator is inserted beneath the muscle to retract the muscle belly laterally. Capsulotomy using scissors starts from the small cut and extends proximally. Once the capsule just medial to the VM insertion and the medial patellofemoral ligament (MPFL) is cut, the patella is moved laterally and the anterior surface of the femur (covered by the ventral part of the fascia and the capsule of the suprapatellar pouch) is exposed, together with a septum that consists of the capsule and the fascia and interposes between the muscle compartment and the joint cavity (Fig. 3B). Then the septum is cut laterally.

\section{Joint Exposure}

The knee is then flexed and the patella is easily dislocated laterally without patellar eversion. If it is still difficult, a proximal extension of the skin or fascia incision can be helpful. The joint is then exposed sufficiently (Fig. 3C). For full access to the proximal tibia, the knee should be flexed maximally. Posterior and lateral retractors are useful to achieve adequate exposure (Fig. 3D).

\section{Closure}

As the capsule has been cut continuously, it can be re- paired in a watertight manner. The end of the capsular incision is located beneath the muscle that is lifted using a hook. A suture is placed from the lateral end of the capsulotomy and continued medially (Fig. 3E). On the medial side of the VM muscle insertion, the capsule becomes thicker and comes closer to the fascia over the VM muscle. This area corresponds to the insertion of the MPFL, so the capsular suture should be made tightly and include the superficial fascial layer (Fig. 3F). Then, the suture is continued downward at the end of the incision.

Next, the skin is lifted and the end of the fasciotomy is found. Another continuous suture is made from the upper end of the capsulotomy and continued downward. At the lower end of the fasciotomy, where the fascia becomes thicker and corresponds to the MPFL insertion, the fascia should be sutured more tightly.

As the quadriceps vectors are retained, good patellar tracking is achieved with the no-thumb technique in most cases. The lateral retinacular release is rarely required if patellar subluxation is not found preoperatively. We do not routinely use a drain. As the capsule is completely repaired in most cases, intra-articular bleeding from the joint does not occur. Early postoperative rehabilitation is possible and no restriction on joint motion is necessary.

We have used this approach since 2009 in more than 1,000 primary knee arthroplasties. No case has required a change to another approach. It virtually has no contraindication and can be used in all primary arthroplasties. It can also be applied to valgus knees with lateral osteoarthritis and to knees with severe contracture. Although
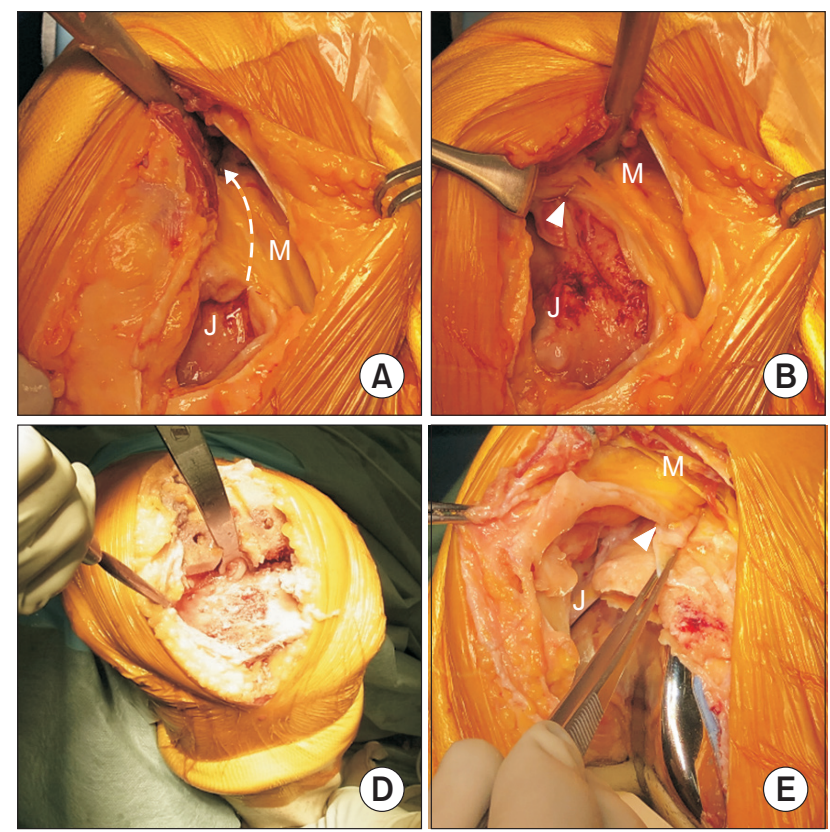

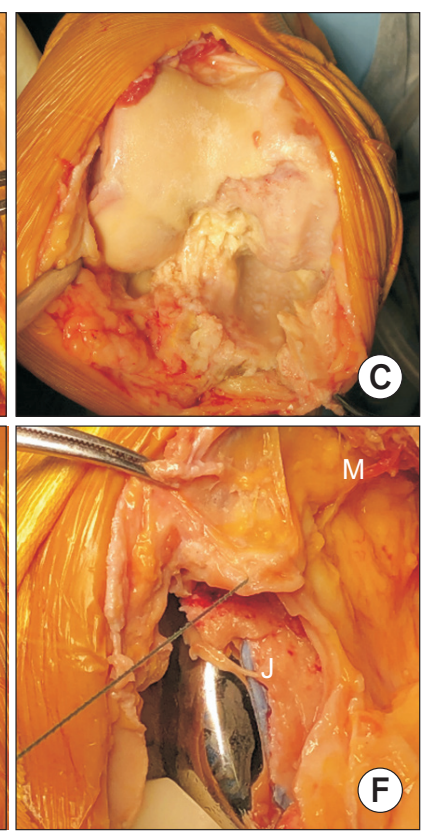

Fig. 3. Surgical steps of the modified under-vastus approach. (A) A capsulotomy is performed under the vastus medialis muscle (broken arrow line). (B) The capsulotomy is complete. The lateral end of the capsulotomy (arrowhead) is visible. (C) Exposure of the femur. Note the muscles are hidden in the skin and are virtually invisible throughout the procedure. (D) Exposure of the tibia. With the posterior and lateral retractors, the tibial cut surface is well exposed. Note the leg is fully flexed by the assistant. (E) At closure, a continuous suture is started at the lateral end of the capsulotomy (arrowhead). (F) The capsule of the suprapatellar pouch is sutured. J: joint cavity of the knee, M: vastus medialis muscle and its compartment. 
very muscular patients are not contraindicated, there were two knees that needed a short VM tendon snip at around $1 \mathrm{~cm}$ in such patients. The snip can be repaired easily and securely by using mattress sutures. There were no intraoperative approach-related complications.

\section{DISCUSSION}

The key feature of the modified UVA is that the capsule is cut transversely and laterally under the VM. Therefore, patellar lateralization is easier than that in the SVA or the original UVA. In the conventional SVA where the capsule is cut medially along the inferior border of the VM, the intact capsule of the suprapatellar pouch that is not elastic can be an obstacle to patellar lateralization. To cut the capsule transversely and laterally, the surgeon must detach the VM from the surroundings. In the original report of UVA by Tatsumi, ${ }^{6}$ the VM was detached along with its fascia. As the detachment is not always easy, the intact fascia itself can limit the elongation of the VM and the patellar lateralization. With our modified UVA, we detach only the muscle belly from the fascia (Fig. $3 \mathrm{~A}$ and $\mathrm{B}$ ) because the muscle belly is elastic and tensile without the fascia. Furthermore, if a better exposure is needed for intraoperative conversion from UKA to TKA, this can be achieved by simple extension of the skin and fascia incision. No further muscular damage is required. Although UKA can be performed through any minimally invasive approach, we prefer to use the UVA for all UKAs since it requires the same but shorter incision. Thus, if a conversion to TKA is needed intraoperatively, only proximal extension of the skin and fascia incision are required.

The second advantage of our modified UVA, compared to other muscle-splitting approaches, is good patellar tracking with less frequent use of a lateral retinacular release..$^{7-9)}$ Lateral retinacular release with the medial parapatellar approach lessens patellar circulation, which may cause anterior knee pain and subsequent patellar fracture. ${ }^{10)}$ However, a randomized comparative study will be necessary to confirm the benefit of the modified UVA over other approaches regarding patellar tracking. This is the key limitation of this paper. Further comparative studies will be necessary to establish conclusively the advantages of this approach.

The third benefit of our modified UVA is the complete capsular repair. This can prevent extra-articular haemorrhage into the muscle. In most cases, cut edges are found without difficulty. After complete repair of the joint capsule, joint fluid and haemorrhage do not flow out. This prevents intramuscular and subcutaneous bleeding that may disturb knee flexion and muscle contraction. Other muscle-retaining approaches such as the SVA and the quadriceps-sparing approach can reduce muscle damage and postoperative muscle pain and weakness. In addition to the benefit of muscle retention, the modified UVA is more beneficial because of capsular repair.

Our modified UVA, a modification of the SVA, is easy to perform. The key element of this approach is detachment of the stretchable muscle belly from surrounding inelastic tissues, which are then incised laterally and repaired completely. Compared to the SVA, the modified UVA allows muscles to be spared, provides better exposure of the operative field, and facilitates anatomical repair of the capsule and fascia. This approach is appropriate for all primary total and unicompartmental knee arthroplasties.

\section{CONFLICT OF INTEREST}

No potential conflict of interest relevant to this article was reported.

\section{REFERENCES}

1. Hofmann AA, Plaster RL, Murdock LE. Subvastus (Southern) approach for primary total knee arthroplasty. Clin Orthop Relat Res. 1991;(269):70-7.

2. Roysam GS, Oakley MJ. Subvastus approach for total knee arthroplasty: a prospective, randomized, and observerblinded trial. J Arthroplasty. 2001;16(4):454-7.

3. Scuderi GR, Tenholder M, Capeci C. Surgical approaches in mini-incision total knee arthroplasty. Clin Orthop Relat Res. 2004;(428):61-7.

4. Tria AJ Jr, Coon TM. Minimal incision total knee ar- throplasty: early experience. Clin Orthop Relat Res. 2003;(416):185-90.

5. Boerger TO, Aglietti P, Mondanelli N, Sensi L. Minisubvastus versus medial parapatellar approach in total knee arthroplasty. Clin Orthop Relat Res. 2005;440:82-7.

6. Tatsumi I. Under vastus approach MIS-TKA. J Minim Invasive Orthop Surg. 2010;54:75-82.

7. Matsueda M, Gustilo RB. Subvastus and medial parapatellar approaches in total knee arthroplasty. Clin Orthop Relat Res. 2000;(371):161-8. 
Hiranaka et al. Modified Under-Vastus Approach for Knee Arthroplasty

Clinics in Orthopedic Surgery • Vol. 11, No. 4, $2019 \bullet$ www.ecios.org

8. Gore DR, Sellinger DS, Gassner KJ, Glaeser ST. Subvastus approach for total knee arthroplasty. Orthopedics. 2003;26(1):33-5.

9. Teng Y, Du W, Jiang J, et al. Subvastus versus medial parapatellar approach in total knee arthroplasty: meta-analysis.
Orthopedics. 2012;35(12):e1722-31.

10. Ritter MA, Pierce MJ, Zhou H, Meding JB, Faris PM, Keating EM. Patellar complications (total knee arthroplasty): effect of lateral release and thickness. Clin Orthop Relat Res. 1999;(367):149-57. 